\title{
Development of Intensity Duration Frequency (IDF) Curves for Rainfall Prediction within the Middle Niger River Basin
}

\author{
Ehiorobo J. O. ${ }^{1 *}$, Izinyon O. C. ${ }^{2}$ and Ilaboya R. I. ${ }^{3}$ \\ ${ }^{1,2,3}$ Department of Civil Engineering, Faculty of Engineering, University of Benin, Benin City, Edo State, \\ Nigeria \\ Corresponding Author: *jacehi@ uniben.edu
}

https://doi.org/10.36263/nijest.2020.02.0231

\begin{abstract}
Rainfall Intensity-Duration-Frequency (IDF) relationship remains one of the mostly used tools in hydrology and water resources engineering, especially for planning, design and operations of water resource projects. IDF relationship can provide adequate information about the intensity of rainfall at different duration for various return periods. The focus of this research was to develop IDF curves for the prediction of rainfall intensity within the middle Niger River Basin (Lokoja and Ilorin) using annual maximum daily rainfall data. Forty (40) year's annual maximum rainfall data ranging from 1974 to 2013 was employed for the study. To ascertain the data quality, selected preliminary analysis technique including; descriptive statistics, test of homogeneity and outlier detection test were employed. To compute the three hours rainfall intensity, the ratio of rainfall amount and duration was used while the popular Gumbel probability distribution model was employed to calculate the rainfall frequency factor. To assess the best fit model that can be employed to predict rainfall intensity for various return periods at ungauged locations, four empirical IDF equations, namely; Talbot, Bernard, Kimijima and Sherman equations were employed. The model with the least calculated sum of minimized root mean square error (RMSE) was adopted as the best fit empirical model. Results obtained revealed that the Talbot model was the best fit model for Ilorin and Lokoja with calculated sum of minimized error of 1.32170E-07 and 8.953636E-08. This model was thereafter employed to predict the rainfall intensity for different durations at 2, 5, 10, 25, 50 and 100yrs return periods respectively.
\end{abstract}

Keywords: Rainfall intensity, Frequency factor, Gumbel probability distribution, Mean and standard deviation

\subsection{Introduction}

Knowledge of intense rainfall characteristics is a useful asset in Engineering application especially for dams dimensioning and design of hydraulic projects (Pruski et al., 2002). Data on intense rainfall characteristics is scarce in most parts of the world especially in the continent of Africa. Even in regions with satisfactory rainfall records, the available data are poor for immediate utilization. Thus, it is necessary to determine the intensity duration- frequency (I-D-F) relations of the intense rainfall (Cardoso et al., 2013). Historical rainfall event statistics (in terms of intensity, duration, and return period) are used to design flood protection structures, and many other civil engineering structures involving hydrologic flows (McCuen, 1998; Prodanovic and Simonovic, 2007). An intensity duration frequency curve is a mathematical model that expresses the relation between intensity, duration, and return period of precipitation. The engineering application of rainfall intensity is mainly in the estimation of design discharge for flood control structures (El-Sayed, 2011; Ugbong, 2000). Generation of intensity duration frequency curves for flood prediction within the Niger River Basin has become imperative owing to the recent devastations caused by flood in various parts within that zone; perhaps being due to the lack of rainfall data and the subsequent design of most drainage structures without appropriate rainfall intensity values (Antigha and Ogharekpe, 2013). Rainfall Intensity-Duration-Frequency (IDF) relationship is one among the numerous tools use in hydrology and water resources engineering since it can provide concise information between the maximum intensity of rain that falls within a given period of time. IDF curves are used in combination with 
runoff estimation formulas such, as the rational method, in order to predict the peak runoff flow from exact point of basin. These are also used in certain aspects of hydraulic structures design such as size of pipes and culvert (Dupont and Allen, 2000).

Attempt has been made by several researchers to develop intensity duration frequency curves in different parts of the world. In a study by Hershfield (1961) various rainfall contour maps were developed to provide the design rain depths for various return periods and durations. Bell (1969) proposed a generalized IDF formula using the one hour, 10 years rainfall depths as an index. Chen (1983) further developed a generalized IDF formula for any location in the United States using three base rainfall depths: one hour 10 years, twenty four hours 10 years and one hour hundred years $\left(P_{l}^{10}\right.$, $P_{24}{ }^{10}, P_{1}{ }^{100}$ ) which describe the geographical variation of rainfall. Antigha and Ogharekpe (2013) developed intensity duration frequency curves for Calabar Metropolis in South-South, Nigeria. In this study, an attempt was made to develop intensity duration frequency curves for Lokaja and Ilorin and also generate models that can be employed to predict the intensity of rainfall for ungauged sites within the study area.

\subsection{Methodology}

\subsection{Description of study area}

The study area is the middle Niger River Basin comprising of Kogi (Lokoja) and parts of Kwara State (Ilorin). Middle Niger Basin system begins at the entry point of River Niger into Nigeria to the outlet into the Gulf of Guinea. The Nigerian part of the basin receives an annual rainfall of between 1,000 and 4,000 $\mathrm{mm}$ (Adeaga et al., 2012) with Inter-annual rainfall variability in the Gulf of Guinea region ranging between 10 to 20 percent. The system has a drainage basin of about $629,545 \mathrm{~km}^{2}$ with discharge contribution of about $117 \mathrm{~km}^{3} /$ year, this constitute about $64.3 \%$ to the total River Niger flow (Clanet, 2009). Flow in the Lower Niger region substantially increases downstream Lokoja after confluence with River Benue, with several tributaries and flows southward before emptying through the Niger Delta, an area characterized by swamps, lagoons, and navigable channels. Mean annual discharge of the Niger upstream of Jebba and downstream of the Kainji and Jebba dams is $1,454 \mathrm{~m}^{3} / \mathrm{s}$. Following the confluence at Lokoja, the flow increases to $5,660 \mathrm{~m}^{3} / \mathrm{s}$ (for the period 1915-2001). Niger River Basin system has a drainage basin of 2,117,700 $\mathrm{km}^{2}$ with annual population growth rate of 3\%, which largely depend on the river for their livelihood. The estimated population is about 104.5 million (80\% of which is in Lower Niger section) and will likely double by 2025 (Clanet, 2009). In addition, over the years the Lower Niger has been experiencing marked decrease in flow with mean flow of $6,055 \mathrm{~m}^{3} / \mathrm{s}\left(191 \mathrm{~km}^{3} /\right.$ year) for $1929-1970$ compared to $5,066 \mathrm{~m}^{3} / \mathrm{s}\left(160 \mathrm{~km}^{3} /\right.$ year $)$ for the period 1971-2001; a decrease of about 17\%. Also recorded is reduction in annual average discharge to about 20\% downstream Kainji Dam before dropping to 45\% (Abam, 2001). Presently, stringent economic pressure through insufficient budget allocation has resulted in varied neglect of water resources assessment infrastructure within the Basin (Adeaga et al., 2012).

\subsection{Data collection}

The data used for this study is annual daily rainfall data obtained from Nigeria Meteorological Agency (NIMET) Abuja. This agency is saddled with the sole responsibility of measuring, analysing and storing meteorological data and forecasting the weather in Nigeria. Daily rainfall data were obtained and analysed with the aid of Microsoft Excel Program in order to determine the annual maximum daily rainfall series (AMDS). To determine the rainfall intensity (I), the popular Gumbel probability distribution model was employed.

\subsection{Preliminary analysis of the data}

Descriptive statistics were used to describe the basic features of the data since they provide simple summaries about the sample and the measures. Together with simple graphics analysis, they form the basis of virtually every quantitative analysis of data. The mean and standard deviation of rainfall intensities were computed as follows:

Mean $=\frac{\sum(I)}{n}$ 
Where:

I Rainfall intensity = Daily Rainfall/24 hrs $(\mathrm{mm} / \mathrm{hr})$

$n \quad$ Number of occurrences

Standard Deviation $=\sqrt{\frac{\sum(I-\bar{I})^{2}}{n-1}}$

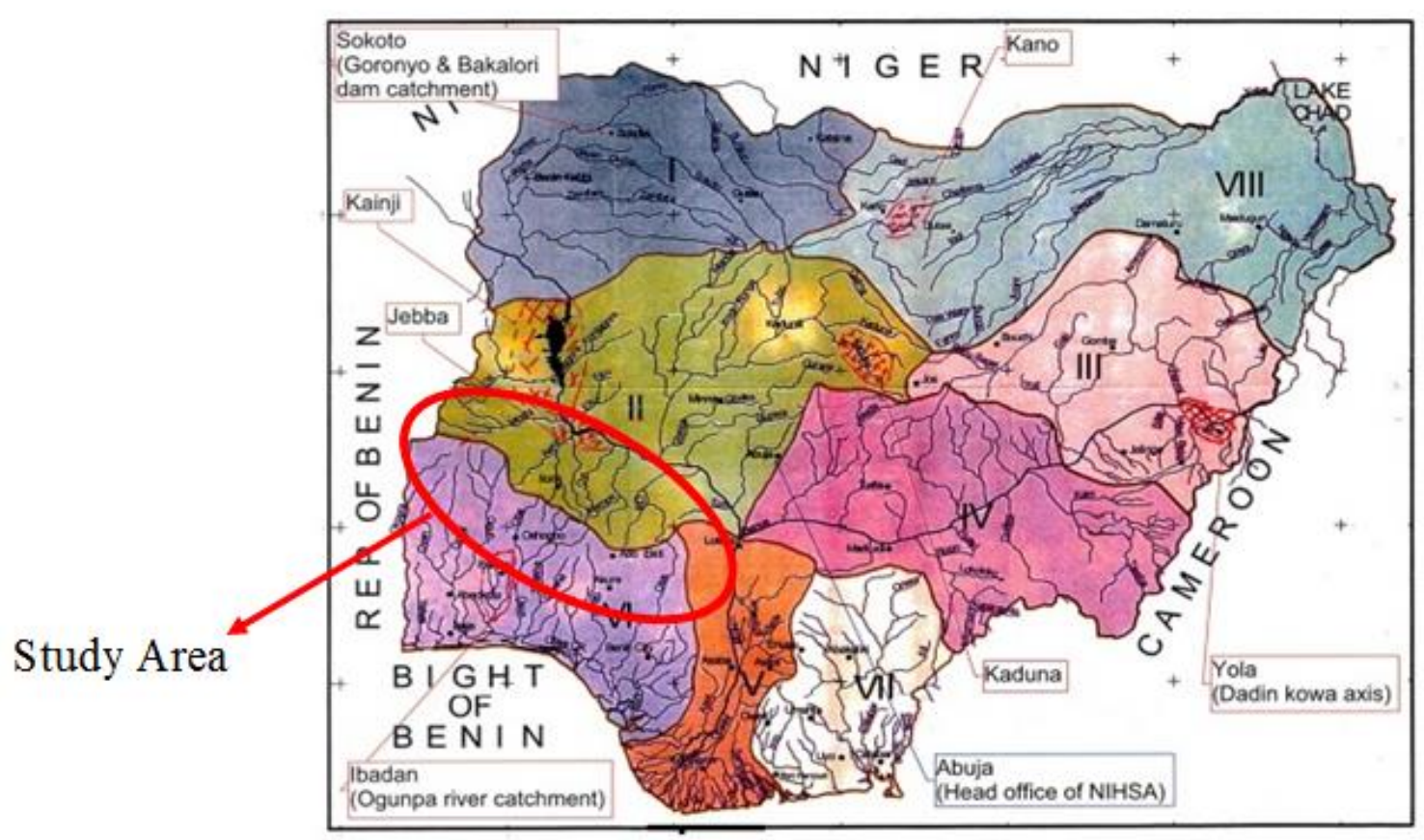

Figure 1: Map of Nigeria showing the study area

\subsubsection{Test of homogeneity}

Owing to the method of data collection coupled with the conditions around the observation site and reliability of the measurement procedures; non-homogeneous observation may appear in the data series and can challenge the quality of the data and the overall outcome of the results since frequency analysis of data requires that the data be homogeneous and independent. Homogeneity test was carried out to establish the fact that the data used are from the same population distribution. The test is based on the cumulative deviation from the mean as expressed using the mathematical equation proposed by Raes et al. (2006) as follows:

$S_{k}=\sum_{i=1}^{k}\left(X_{i}-\bar{X}\right) k=1, \ldots . n$

\footnotetext{
Where:

$X_{i} \quad$ The record for the series $\mathrm{X}_{1}, \mathrm{X}_{2} \ldots \mathrm{X}_{\mathrm{n}}$

$\bar{X} \quad$ The mean

$S_{k s} \quad$ The residual mass curve
}

For a homogeneous record, it is expected that the data points fluctuate around zero line as defined by the residual mass curve. To perform the homogeneity test, a software package (Rainbow) for analysing hydrological data was employed. 
To further confirm that the rainfall data are statistically homogeneous, test of hypothesis was done using test of hypothesis. The null hypothesis of homogeneity was formulated as follows:

$\mathrm{H}_{0}$ : Data are statistically homogeneous

$\mathrm{H}_{1}$ : Data are not homogeneous

The null and alternate hypotheses were tested at $90 \%, 95 \%$ and $99 \%$ confidence interval that is 0.1 , 0.05 and 0.01 degree of freedom respectively.

\subsubsection{Outlier detection}

Outliers are defined as data point that split off or are very different from the rest of the data (Stevens, 1986). Outliers can be caused by irregularities or errors which occur during the data recording or observation process. These points deserve further investigation in order to decide whether or not to remove them. The problem of outliers is of major concern when dealing with extreme events. Although, numerous methods abound in the literature for the analysis and determination of outliers, in this study, the labelling rule method was employed to detect the presence of outliers. The labelling rule is the statistical method of detecting the presence of outliers in a data set using the 25th percentile (lower bound) and the 75th percentile (upper bound). The underlying mathematical equation based on the lower and the upper bound as proposed by Levi et al. (2009) is presented as follows:

Lower Bound

$$
Q_{1}-\left[2.2 \times\left(Q_{3}-Q_{1}\right)\right]
$$

Upper Bound

$$
Q_{3}+\left[2.2 \times\left(Q_{3}-Q_{1}\right)\right]
$$

At 0.05 degree of freedom, any data lower than $\mathrm{Q}_{1}$ or greater than $\mathrm{Q}_{3}$ was considered an outlier and was removed before the analysis.

\subsubsection{Generation of IDF curve}

Rainfall intensity is defined as the ratio of the total amount of rain (rainfall depth) falling during a given period to the duration of the period It is expressed in depth units per unit time, usually as $\mathrm{mm}$ per hour $(\mathrm{mm} / \mathrm{hr})$. The step by step procedure for generating the IDF curves is as follows:

i. Annual maximum daily series of rainfall were obtained from the daily rainfall data

ii. The descriptive statistics of the data consisting; the mean $(\overline{\mathrm{X}})$ and standard deviation $(\mathrm{S})$ were computed using equations (2.1) and (2.2) respectively.

iii. The three hours rainfall intensity was computed using:

$$
I=\frac{R}{T}
$$

Where; $I$ is the rainfall intensity $(\mathrm{mm} / \mathrm{hr}), R$ is the amount of rainfall $(\mathrm{mm})$ and $T$ is the duration of the rainfall ( $3 \mathrm{hrs}$ ).

iv. The rainfall frequency factor $\left(\mathrm{K}_{\mathrm{T}}\right)$ for selected return periods $(\mathrm{T}=2,5,10,25,50$ and 100yrs) were computed using the quantile equation of the Gumbel probability distribution model and is presented as follows:

$$
K_{T}=-\frac{\sqrt{6}}{\pi}\left\langle 0.5772+\ln \left[\ln \left(\frac{T}{T-1}\right)\right]\right\rangle
$$

Where; $T$ is the selected return periods

v. The rainfall intensity corresponding to a specified return period was computed using:

$X_{T}=\bar{X}+K_{T} S$

Where; $X_{T}$ is the rainfall intensity corresponding to a specified return period $T$ (yrs), $\overline{\mathrm{X}}$ is the average of the maximum precipitation corresponding to a specific duration and $S$ is the standard deviation of precipitation data. 
vi. Finally, the IDF curves were generated from the plot of rainfall intensities against duration for corresponding return period.

\subsection{Results and Discussion}

Descriptive statistics of annual maximum daily rainfall data employed for this analysis is presented in

Tables 1 and 2 respectively.

Table 1: Descriptive statistics of AMDS from Lokoja

\begin{tabular}{||ll|r|r||}
\hline & & \multicolumn{1}{|c|}{ Statistic } & Std. Error \\
\hline LOKOJA & Mean & 79.315 & 3.2721 \\
$95 \%$ Confidence Interval & Lower Bound & 72.732 & \\
for Mean & Upper Bound & 85.897 & \\
5\% Trimmed Mean & & 77.554 & \\
Median & 72.500 & \\
Variance & 513.912 & \\
Std. Deviation & 22.6696 & \\
Minimum & 49.5 & \\
Maximum & 174.2 & \\
Range & 124.7 & \\
Interquartile Range & 32.6 & \\
Skewness & 1.696 & .343 \\
Kurtosis & 5.015 & .674 \\
\hline
\end{tabular}

Table 2: Descriptive statistics of AMDS from Ilorin

\begin{tabular}{|c|c|c|c|c|}
\hline ILORIN & $\begin{array}{l}\text { Mean } \\
95 \% \text { Confidence Interval } \\
\text { for Mean } \\
5 \% \text { Trimmed Mean } \\
\text { Median } \\
\text { Variance } \\
\text { Std. Deviation } \\
\text { Minimum } \\
\text { Maximum } \\
\text { Range } \\
\text { Interquartile Range } \\
\text { Skewness } \\
\text { Kurtosis }\end{array}$ & $\begin{array}{l}\text { Lower Bound } \\
\text { Upper Bound }\end{array}$ & $\begin{array}{r}79.352 \\
72.415 \\
86.289 \\
77.770 \\
76.000 \\
570.776 \\
23.8909 \\
36.6 \\
160.5 \\
123.9 \\
24.7 \\
1.137 \\
2.113\end{array}$ & 3.4484 \\
\hline
\end{tabular}

Results of the homogeneity test conducted on the annual maximum daily rainfall series for the stations employed in the study and presented in Figures $2 \mathrm{a}$ and $2 \mathrm{~b}$ revealed that the data used are statistically homogeneous. Results of homogeneity statistics employed to test the strength of the null hypothesis over the alternate hypothesis is presented in Figures $3 \mathrm{a}$ and $3 \mathrm{~b}$.
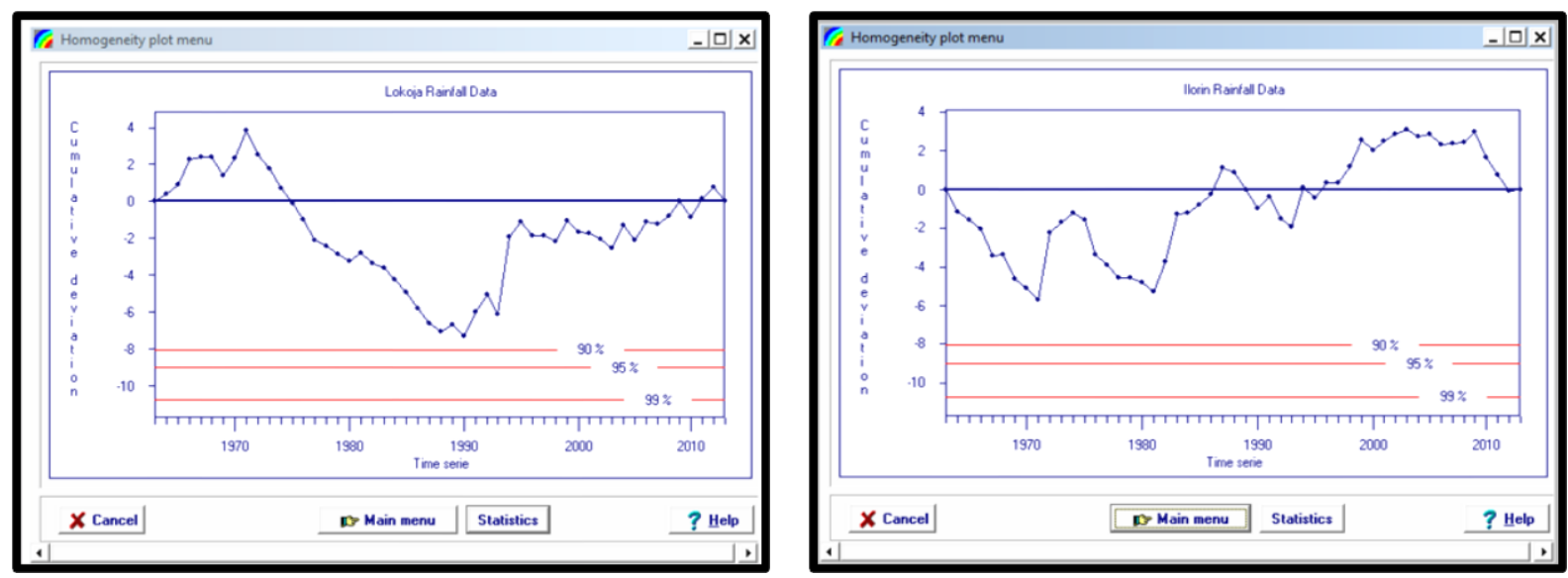
Figure 2a: Homogeneity test of Lokoja AMDS

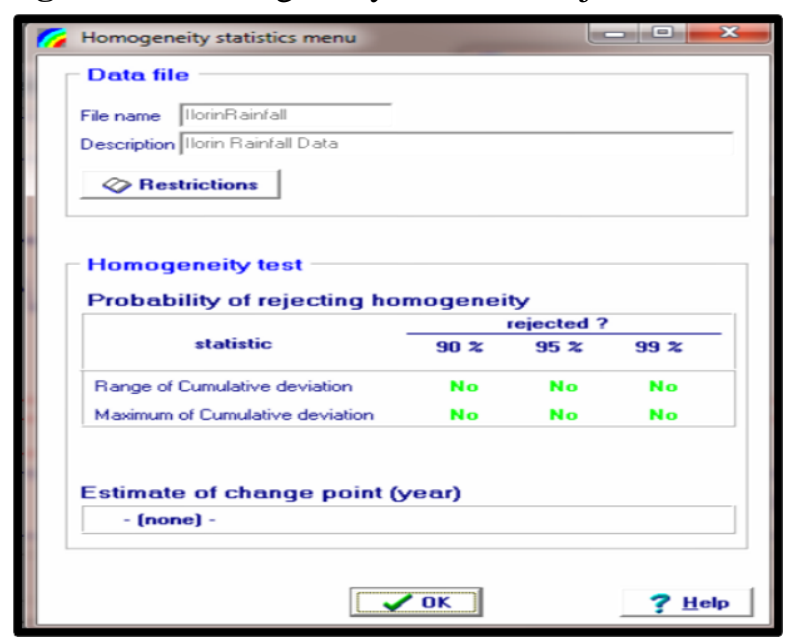

Figure 3a: Homogeneity statistics of Lokoja AMDS
Figure 2a: Homogeneity test of Ilorin AMDS

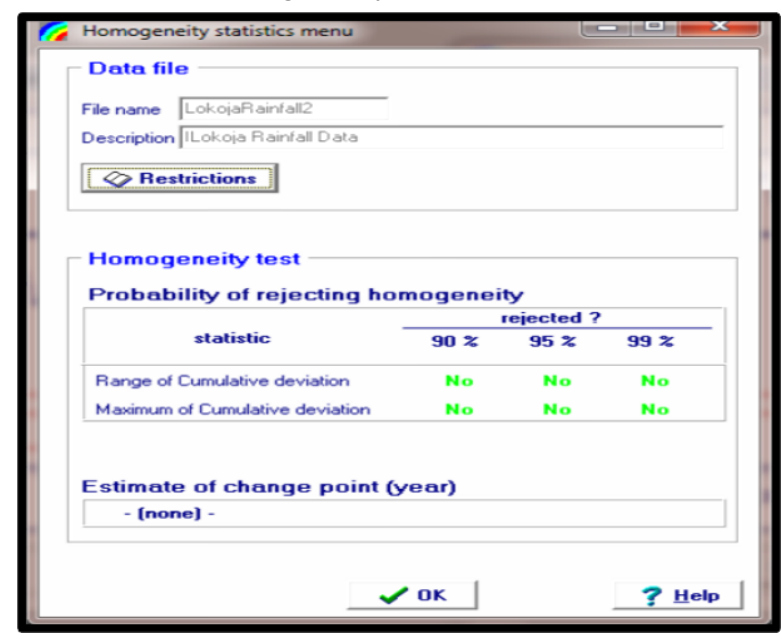

Figure 3b: Homogeneity statistics of Ilorin AMDS

Based on the computed statistics as presented in Figures $3 \mathrm{a}$ and $3 \mathrm{~b}$, the null hypothesis $\left(\mathrm{H}_{0}\right)$ was accepted, and it was concluded that the rainfall data collected from Lokoja and Ilorin are statistically homogeneous at $90 \%, 95 \%$ and $99 \%$ confidence interval that is $0.10,0.05$ and $0.01 \mathrm{df}$. The computed rainfall percentiles presented in Table 3 was employed to analyse and determine the presence of outlier in the data.

Table 3: Computed rainfall percentiles

\begin{tabular}{|l|c|c|c|c|c|c|c|c|}
\hline \multicolumn{10}{|c|}{ Percentiles } \\
\hline & & \multicolumn{7}{|c|}{ Percentiles } \\
\cline { 3 - 9 } & 5 & 10 & 25 & 50 & 75 & 90 & 95 \\
\hline Meighted Average & LOKOJA & 54.180 & 56.300 & 62.100 & 72.500 & 94.750 & 107.420 & 112.010 \\
(Definition 1) & ILORIN & 46.780 & 52.020 & 65.925 & 76.000 & 90.625 & 111.890 & 132.055 \\
\hline Tukey's Hinges & LOKO.JA & & & 62.200 & 72.500 & 93.200 & & \\
& ILORIN & & & 66.050 & 76.000 & 90.550 & & \\
\hline
\end{tabular}

Using the weighted average definition, the 25th percentile $\left(\mathrm{Q}_{1}\right)$ of AMDS from Lokoja was observed to be 62.10 while the 75 th percentile $\left(\mathrm{Q}_{3}\right)$ was observed to be 94.75 . Adopting the labelling rule equation, the lower and upper bound statistics of AMDS from Lokoja were calculated as follows:

Lower bound $=62.10-(2.2(94.75-62.10))=-9.73$

Upper bound $=94.75+(2.2(94.75-62.10))=166.58$

The 25th percentile $\left(\mathrm{Q}_{1}\right)$ of AMDS from Ilorin was observed to be 65.925 while the 75 th percentile $\left(\mathrm{Q}_{3}\right)$ was observed to be 90.625 . Adopting the labelling rule equation, the lower and upper bound statistics of AMDS from Ilorin were also calculated as follows:

Lower bound $=65.925-(2.2(90.625-65.925))=-11.585$

Upper bound $=90.625+(2.2(90.625-65.925))=144.965$

The extreme value statistics of AMDS from Lokoja and Ilorin which shows the highest and lowest case numbers are presented in Tables $4 \mathrm{a}$ and $4 \mathrm{~b}$ respectively. From the result of Table $4 \mathrm{a}$, it was observed that the highest AMDS from Lokoja is 174.20, which is higher than the calculated upper bound of 166.58. The lowest AMDS was observed to be 49.50, which is greater than the calculated lower bound of -9.73 . To be devoid of outlier, no rainfall value must be greater than the calculated upper bound or lower than the calculated lower bound. AMDS of $174.20 \mathrm{~mm}$ represented by case number 31 was declared an outlier since it is greater than the calculated upper bound of 166.58 and 
was removed before further analysis. From the result of Table $4 \mathrm{~b}$, it was observed that the highest AMDS from Ilorin is 160.50, which is higher than the calculated upper bound of 144.965. The lowest AMDS was observed to be 36.60 which is greater than the calculated lower bound of -11.585 . To be devoid of outlier, no rainfall value must be greater than the calculated upper bound or lower than the calculated lower bound. AMDS of $160.50 \mathrm{~mm}$ represented by case number 9 was declared an outlier since it is greater than the calculated upper bound of 144.965 and was also removed before further analysis. A further test of outliers was done using the box plots presented in Figures $4 \mathrm{a}$ and $4 \mathrm{~b}$ respectively.

Table 4a: Extreme value statistics of AMDS from Lokoja

\begin{tabular}{|c|c|c|c|c|}
\hline & & & Case Number & Value \\
\hline \multirow[t]{10}{*}{ LOKOJA } & \multirow[t]{5}{*}{ Highest } & 1 & 31 & 174.2 \\
\hline & & 2 & 8 & 113.0 \\
\hline & & 3 & 3 & 110.8 \\
\hline & & 4 & 28 & 108.5 \\
\hline & & 5 & 41 & 107.3 \\
\hline & \multirow[t]{5}{*}{ Lowest } & 1 & 9 & 49.5 \\
\hline & & 2 & 14 & 54.0 \\
\hline & & 3 & 30 & 54.4 \\
\hline & & 4 & 11 & 55.4 \\
\hline & & 5 & 6 & 56.4 \\
\hline
\end{tabular}

Table 4b: Extreme value statistics of AMDS from Ilorin

\begin{tabular}{|rrr|r|r|}
\hline \hline ILORIN & Highest & 1 & 9 & 160.5 \\
& & 20 & 136.6 \\
& & 31 & 126.5 \\
& 3 & 19 & 115.4 \\
& 4 & 36 & 111.5 \\
\cline { 2 - 4 } & & 13 & 36.6 \\
& Lowest & 1 & 4 & 45.7 \\
& 2 & 47 & 48.1 \\
& 3 & 6 & 49.5 \\
& 4 & 1 & 52.3 \\
\hline
\end{tabular}

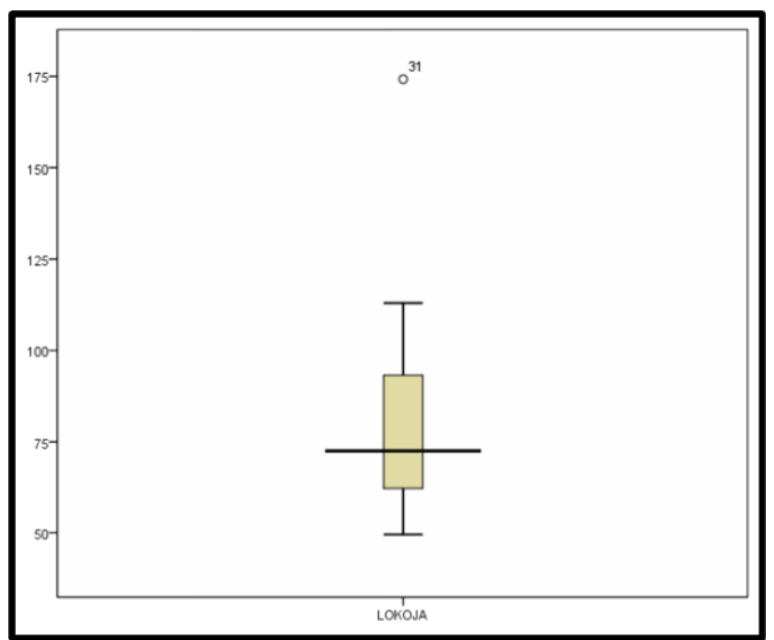

Figure 4a: Box plot of AMDS from Lokoja

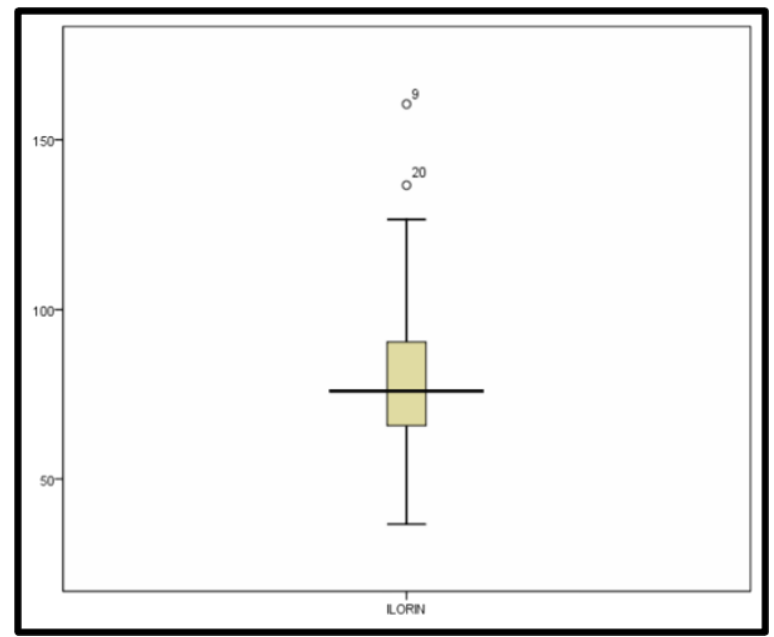

Figure 4b: Box plot of AMDS from Ilorin

It was concluded based on the results of Figures $4 \mathrm{a}$ and $4 \mathrm{~b}$ that AMDS represented by case numbers 31 for Lokoja and 9 for Ilorin are outliers and were removed before further analysis. Using the final data and employing the procedures based on the Gumbel probability distribution model outlined for the calculation of rainfall intensity, the calculated rainfall intensities for Ilorin and Lokoja for the selected return periods is presented in Tables $5 \mathrm{a}$ and $5 \mathrm{~b}$ respectively. Using the computed rainfall intensity, intensity duration frequency, (IDF) curves were generated and are presented in Figures 5a, $5 b, 5 c$ and $6 a, 6 b$ and $6 c$ respectively. 
Table 5a: Calculated Rainfall Intensity for Ilorin based on Gumbel distribution

\begin{tabular}{|c|c|c|c|c|c|c|}
\hline $\begin{array}{c}\text { Duration } \\
(\mathrm{hrs})\end{array}$ & $\begin{array}{c}\text { Intensity } \\
(\mathrm{mm} / \mathrm{hr}) \\
(\mathrm{T}=2 \mathrm{yrs})\end{array}$ & $\begin{array}{c}\text { Intensity } \\
(\mathrm{mm} / \mathrm{hr}) \\
(\mathrm{T}=5 \mathrm{yrs})\end{array}$ & $\begin{array}{c}\text { Intensity } \\
(\mathrm{mm} / \mathrm{hr}) \\
(\mathrm{T}=10 \mathrm{yrs})\end{array}$ & $\begin{array}{c}\text { Intensity } \\
(\mathrm{mm} / \mathrm{hr}) \\
(\mathrm{T}=25 \mathrm{yrs})\end{array}$ & $\begin{array}{c}\text { Intensity } \\
(\mathrm{mm} / \mathrm{hr}) \\
(\mathrm{T}=50 \mathrm{yrs})\end{array}$ & $\begin{array}{c}\text { Intensity }(\mathrm{mm} / \mathrm{hr}) \\
(\mathrm{T}=100 \mathrm{yrs})\end{array}$ \\
\hline 0.0333 & 755.0297719 & 966.3721533 & 1106.299199 & 1283.097393 & 1414.256392 & 1544.446935 \\
\hline 0.0833 & 301.8306291 & 386.3168392 & 442.2540616 & 512.9308905 & 565.3629996 & 617.4079582 \\
\hline 0.1667 & 150.8247835 & 193.0425477 & 220.9943811 & 256.3115967 & 282.5119248 & 308.5187938 \\
\hline 0.25 & 100.5699656 & 128.7207708 & 147.3590533 & 170.9085727 & 188.3789515 & 205.7203317 \\
\hline 0.5 & 50.28498281 & 64.36038541 & 73.67952667 & 85.45428635 & 94.18947573 & 102.8601658 \\
\hline 1 & 25.14249141 & 32.1801927 & 36.83976333 & 42.72714318 & 47.09473787 & 51.43008292 \\
\hline 2 & 12.5712457 & 16.09009635 & 18.41988167 & 21.36357159 & 23.54736893 & 25.71504146 \\
\hline 3 & 8.380830469 & 10.7267309 & 12.27992111 & 14.24238106 & 15.69824596 & 17.14336097 \\
\hline 4 & 6.285622851 & 8.045048176 & 9.209940833 & 10.68178579 & 11.77368447 & 12.85752073 \\
\hline 5.333 & 4.714511796 & 6.034163267 & 6.907887368 & 8.011840086 & 8.830815276 & 9.643743282 \\
\hline
\end{tabular}

Table 5b: Calculated Rainfall Intensity for Lokoja based on Gumbel distribution

\begin{tabular}{|c|c|c|c|c|c|c|}
\hline $\begin{array}{c}\text { Duration } \\
(\mathrm{hrs})\end{array}$ & $\begin{array}{c}\text { Intensity } \\
(\mathrm{mm} / \mathrm{hr}) \\
(\mathrm{T}=2 \mathrm{yrs})\end{array}$ & $\begin{array}{c}\text { Intensity } \\
(\mathrm{mm} / \mathrm{hr}) \\
(\mathrm{T}=5 \mathrm{yrs})\end{array}$ & $\begin{array}{c}\text { Intensity } \\
(\mathrm{mm} / \mathrm{hr}) \\
(\mathrm{T}=10 \mathrm{yrs})\end{array}$ & $\begin{array}{c}\text { Intensity } \\
(\mathrm{mm} / \mathrm{hr}) \\
(\mathrm{T}=25 \mathrm{yrs})\end{array}$ & $\begin{array}{c}\text { Intensity } \\
(\mathrm{mm} / \mathrm{hr}) \\
(\mathrm{T}=50 \mathrm{yrs})\end{array}$ & $\begin{array}{c}\text { Intensity }(\mathrm{mm} / \mathrm{hr}) \\
(\mathrm{T}=100 \mathrm{yrs})\end{array}$ \\
\hline 0.0333 & 756.6626242 & 957.2014025 & 1089.975523 & 1257.735977 & 1382.190265 & 1505.725602 \\
\hline 0.0833 & 302.483378 & 382.6507408 & 435.7285105 & 502.7924133 & 552.5442474 & 601.9287219 \\
\hline 0.1667 & 151.1509621 & 191.2105981 & 217.7335628 & 251.2453991 & 276.1063936 & 300.7838184 \\
\hline 0.25 & 100.7874615 & 127.4992268 & 145.1847397 & 167.5304321 & 184.1077432 & 200.5626501 \\
\hline 0.5 & 50.39373077 & 63.74961341 & 72.59236985 & 83.76521605 & 92.05387162 & 100.2813251 \\
\hline 1 & 25.19686539 & 31.8748067 & 36.29618492 & 41.88260803 & 46.02693581 & 50.14066253 \\
\hline 2 & 12.59843269 & 15.93740335 & 18.14809246 & 20.94130401 & 23.01346791 & 25.07033127 \\
\hline 3 & 8.398955129 & 10.62493557 & 12.09872831 & 13.96086934 & 15.34231194 & 16.71355418 \\
\hline 4 & 6.299216346 & 7.968701676 & 9.074046231 & 10.47065201 & 11.50673395 & 12.53516563 \\
\hline 5.333 & 4.724707554 & 5.976899813 & 6.805960046 & 7.853479847 & 8.630589876 & 9.401961847 \\
\hline
\end{tabular}

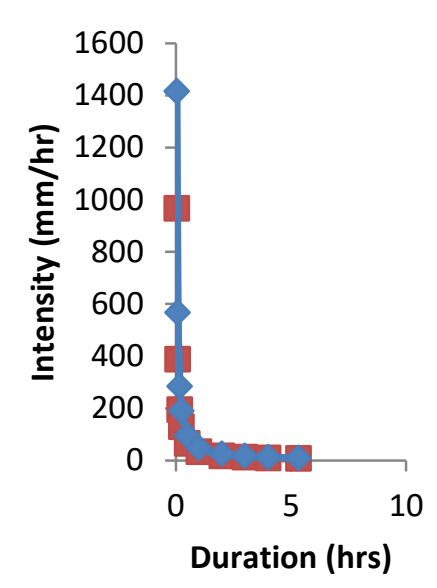

Figure 5a: IDF curves for Ilorin based on Gumbel distribution (5 and 50 yrs return period)

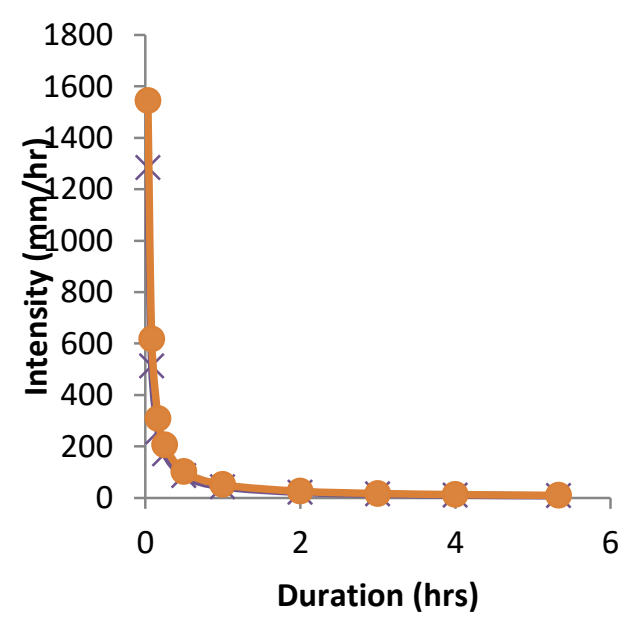

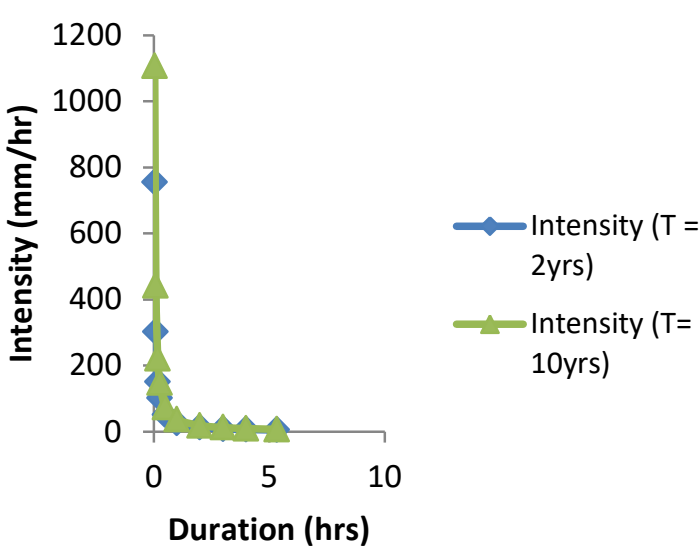

Figure 5b: IDF curves for Ilorin based on Gumbel distribution ( 2 and 10 yrs return period)

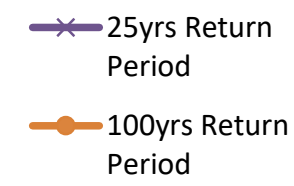

6

Figure 5c: IDF curves for Ilorin based on Gumbel distribution (25 and 100 yrs return period) 


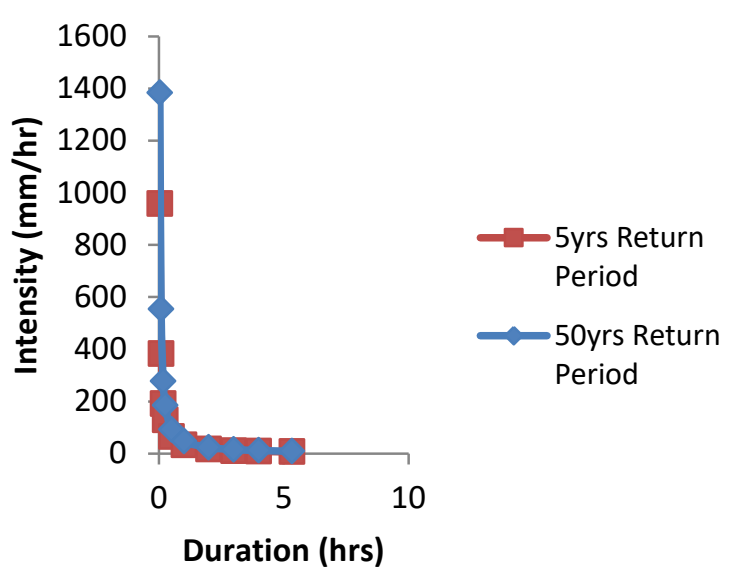

Figure 6a: IDF curves for Lokoja based on Gumbel distribution (5 and 50 yrs return period)

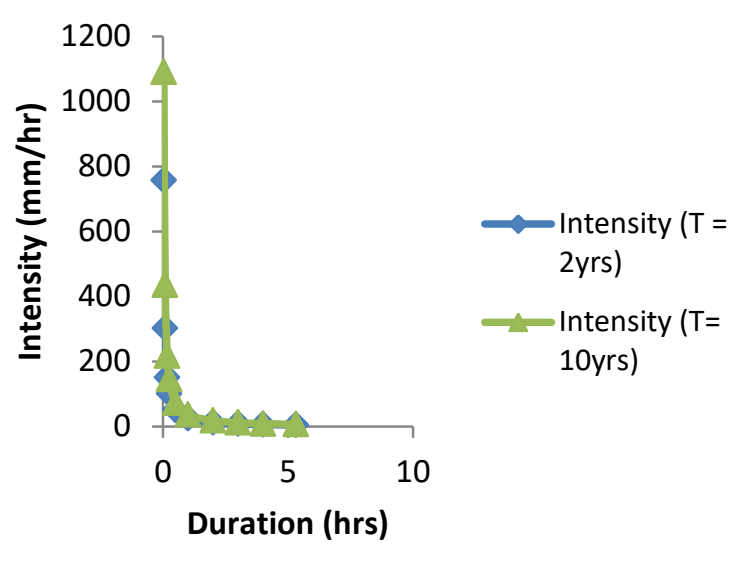

Figure 6b: IDF curves for Lokoja based on Gumbel distribution (2 and 10 yrs return period)

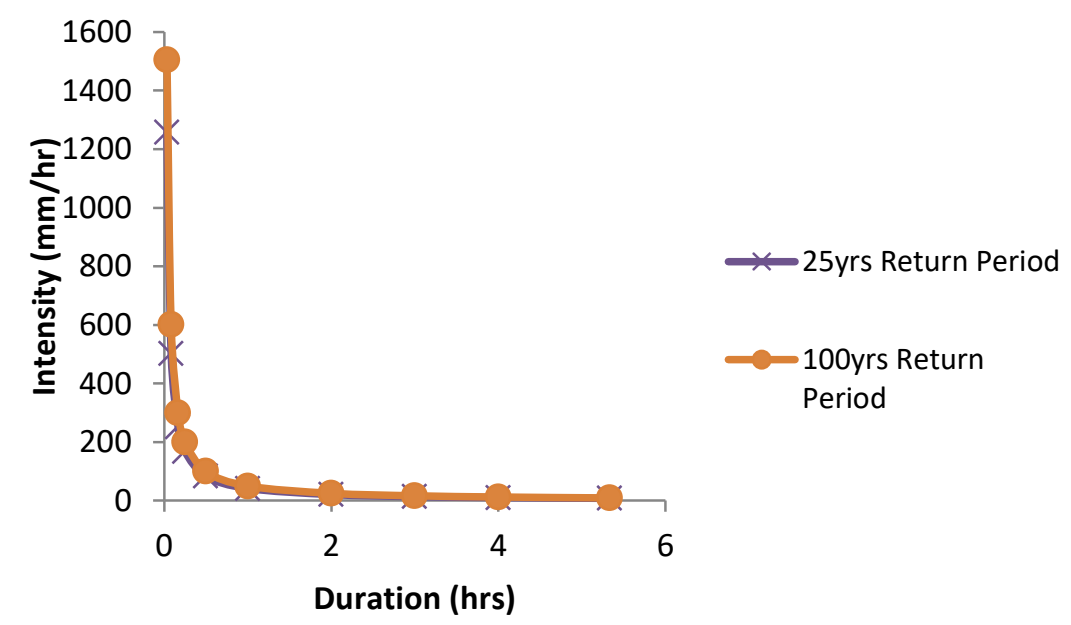

Figure 6c: IDF curves for Ilorin based on Gumbel distribution (25 and 100 yrs return period)

\subsection{Fitting of empirical IDF equations}

Empirical IDF equations represent the relationship between maximum rainfall intensity (as dependent variable) and other parameters of interest such as rainfall duration and frequency (as independent variables). There are several commonly used equations presented in literature for hydrology applications, presented by (Chow et al., 1988), used for the analysis of rainfall intensity. For this study, four basic forms of equations used to describe the rainfall intensity duration relationship presented in Table 6 were employed.

Table 6: Definition of selected empirical rainfall intensity equations

\begin{tabular}{|l|l|l|}
\hline S/N & Empirical Equation & Reference \\
\hline 1 & $i=\frac{a}{d+b}$ & Talbot Equation \\
\hline 2 & $i=\frac{a}{d^{e}}$ & Bernard Equation \\
\hline 3 & $i=\frac{a}{d^{e}+b}$ & Kimijima Equation \\
\hline 4 & $i=\frac{a}{(d+b)^{e}}$ & Sherman Equation \\
\hline
\end{tabular}

where:

$\begin{array}{ll}i & \text { Rainfall intensity (mm/hr); } \\ d & \text { Duration (hrs); }\end{array}$




\section{$a, b$ and $e \quad$ Constant parameters related to the empirical equation.}

These empirical equations show that rainfall intensity decreases with rainfall duration for a given return period. To determine the parameters of the equation, non-linear regression techniques using the Microsoft excel solver was employed. The inter phase of the non-linear regression solver is presented in Tables 7, 8, 9 and 10 respectively.

Table 7: Non-linear regression solver based on Talbot equation

\begin{tabular}{|c|c|c|c|c|c|c|c|}
\hline A & B & C & D & E & $\mathrm{F}$ & G & H \\
\hline d & i & Talbot Equation & Upper Cl & Lower Cl & & a & 25.5493397485865 \\
\hline 0.0333 & 755.029771937681 & $=(\$ H \$ 1) /(A 2+\$ H \$ 2)$ & $=\mathrm{C} 2+\$ \mathrm{H} \$ 8$ & $=\mathrm{C} 2-\$ \mathrm{H} \$ 8$ & & b & 0.00809089069609902 \\
\hline 0.0833 & 301.830629117945 & $=(\$ H \$ 1) /(A 3+\$ H \$ 2)$ & $=\mathrm{C} 3+\$ \mathrm{H} \$ \mathbf{8}$ & $=C 3-\$ H \$ 8$ & & Mean of $i$ & $=$ AVERAGE(B2:B11) \\
\hline 0.1667 & 150.824783476453 & $=(\$ H \$ 1) /(A 4+\$ H \$ 2)$ & $=C 4+\$ \$ \$ \$ B$ & $=C 4-\$ \$ H \$ S$ & & df & $=\operatorname{COUNT}(\mathrm{B} 2: \mathrm{B} 11)-\operatorname{COUNT}(\mathrm{H} 1: \mathrm{H} 2)$ \\
\hline 0.25 & 100.569965622099 & $=(\$ H \$ 1) /(A 5+\$ H \$ 2)$ & $=\mathrm{C} 5+\$ \$ \mathrm{H} \$ \mathbf{8}$ & $=\mathrm{C} 5-\$ \mathrm{H} \$ 8$ & & SSE & $=$ SQRT $\left(\mathrm{SUM}\left((\mathrm{B} 2: \mathrm{B} 11-\mathrm{C2}: \mathrm{C} 11)^{\wedge} 2\right) / \mathrm{H} 4\right)$ \\
\hline 0.5 & 50.2849828110496 & $=(\$ H \$ 1) /(A 6+\$ H \$ 2)$ & $=C 6+\$ \$ \$ 8$ & $=C 6-\$ H \$ 8$ & & $\mathrm{R}^{2}$ & $=1-\mathrm{SUM}\left((\mathrm{B} 2: \mathrm{B} 11-\mathrm{C} 2: \mathrm{C} 11)^{\wedge} 2\right) / \mathrm{SUM}\left((\mathrm{B} 2: B 11-\mathrm{H} 3)^{\wedge} 2\right)$ \\
\hline 1 & 25.1424914055248 & $=(\$ H \$ 1) /(A 7+\$ H \$ 2)$ & $=\mathrm{C} 7+\$ \mathrm{H} \$ \mathbf{8}$ & $=C 7-\$ H \$ 8$ & & Critical t & $=\operatorname{TINV}(0.05, \mathrm{H} 4)$ \\
\hline 2 & 12.5712457027624 & $=(\$ H \$ 1) /(A 8+\$ H \$ 2)$ & $=C 8+\$+\$ \$ 8$ & $=C 8-\$ \$ H \$ B$ & & $\mathrm{Cl}$ & $=\left(\mathrm{H} 7^{*} \mathrm{H} 5\right)$ \\
\hline 3 & 8.38083046850826 & $=(\$ H \$ 1) /(A 9+\$ H \$ 2)$ & $=C 9+\$ \mathrm{H} \$ \mathrm{~B}$ & $=C 9-\$ H \$ 8$ & & HYBRID & $=100^{*}\left(\operatorname{SUM}\left(\left((\mathrm{B} 2: \mathrm{B} 11-\mathrm{C} 2: \mathrm{C} 11)^{\wedge} 2\right) /(\mathrm{B} 2: \mathrm{B} 11)\right)\right) /(\operatorname{COUNT}(\mathrm{B} 2: \mathrm{B} 11)-1)$ \\
\hline 4 & 6.28562285138119 & $=(\$ H \$ 1) /(A 10+\$ H \$ 2)$ & $=\mathrm{C} 10+\$ \mathrm{H} \$ 8$ & $=\mathrm{C} 10-\$ \mathrm{H} \$ 8$ & & NSD & $\left.\left.=100^{*}\left(\operatorname{SQRT}(\mathrm{SUM})((\mathrm{B} 2: \mathrm{B} 11-\mathrm{C} 2: \mathrm{C} 11) /(\mathrm{B} 2: \mathrm{B} 11))^{\wedge} 2\right) /(\operatorname{COUNT}(\mathrm{B} 2: \mathrm{B} 11)-1)\right)\right)$ \\
\hline 5.333 & 4.71451179552312 & $=(\$ H \$ 1) /(A 11+\$ H \$ 2)$ & $=C 11+\$ H \$ 8$ & $=C 11-\$ H \$ 8$ & & RMSE & $=$ SQRT(SUM $\left.\left((B 2: B 11-C 2: C 11)^{\wedge} 2\right) /(C O U N T(B 2: B 11)-1)\right)$ \\
\hline
\end{tabular}

Table 8: Non-linear regression solver based on Bernard equation

\begin{tabular}{|c|c|c|c|c|c|c|c|}
\hline A & B & C & D & E & $\mathrm{F}$ & G & H \\
\hline d & i & BERNARD EQUATION & Upper Cl & Lower Cl & & a & 4.73963937022917 \\
\hline 0.0333 & 755.029771937681 & $=(\$ H \$ 1) /((A 2) \wedge \$ H \$ 2)$ & $=\mathrm{C} 2+\$ \mathrm{H} \$ 8$ & $=\mathrm{C} 2-\$ \mathrm{H} \$ 8$ & & e & 0.00317555486873956 \\
\hline 0.0833 & 301.830629117945 & $=(\$ H \$ 1) /\left((A 3)^{\wedge} \$ H \$ 2\right)$ & $=\mathrm{C} 3+\$ \mathrm{H} \$ 8$ & $=\mathrm{C} 3-\$ \mathrm{H} \$ \$ 8$ & & Mean of $i$ & $=$ AVERAGE(B2:B11) \\
\hline 0.1667 & 150.824783476453 & $=(\$ H \$ 1) /\left((A 4)^{\wedge} \$ H \$ 2\right)$ & $=C 4+\$ H \$ 8$ & $=C 4-\$ H \$ 8$ & & df & $=\operatorname{COUNT}(\mathrm{B} 2: \mathrm{B} 11)-\operatorname{COUNT}(\mathrm{H1}: \mathrm{H} 2)$ \\
\hline 0.25 & 100.569965622099 & $=(\$ H \$ 1) /\left((A 5)^{\wedge} \$ \mathrm{H} \$ 2\right)$ & $=\mathrm{C} 5+\$ \mathrm{H} \$ 8$ & $=$ C5-\$\$H\$8 & & SSE & $=\operatorname{SQRT}\left(\mathrm{SUM}\left((\mathrm{B} 2: \mathrm{B} 11-\mathrm{C} 2: \mathrm{C} 11)^{\wedge} 2\right) / \mathrm{H} 4\right)$ \\
\hline 0.5 & 50.2849828110496 & $=(\$ H \$ 1) /\left((A 6)^{\wedge} \$ H \$ 2\right)$ & $=\mathrm{C} 6+\$ \mathrm{H} \$ 8$ & $=\mathrm{C} 6-\$ \mathrm{H} \$ 8$ & & $R^{2}$ & $=1-\mathrm{SUM}\left((\mathrm{B} 2: \mathrm{B} 11-\mathrm{C} 2: \mathrm{C} 11)^{\wedge} 2\right) / \mathrm{SUM}\left((\mathrm{B} 2: \mathrm{B} 11-\mathrm{H} 3)^{\wedge} 2\right)$ \\
\hline 1 & 25.1424914055248 & $=(\$ H \$ 1) /((A 7) \wedge \$ H \$ 2)$ & $=\mathrm{C} 7+\$ \mathrm{H} \$ 8$ & $=\mathrm{C} 7-\$ \mathrm{H} \$ 8$ & & Critical t & $=\operatorname{TINV}(0.05, \mathrm{H} 4)$ \\
\hline 2 & 12.5712457027624 & $=(\$ H \$ 1) /\left((A 8)^{\wedge} \$ H \$ 2\right)$ & $=\mathrm{C} 8+\$ \mathrm{H} \$ 8$ & $=C 8-\$ H \$ 8$ & & $\mathrm{Cl}$ & $=\left(H 7^{*} H 5\right)$ \\
\hline 3 & 8.38083046850826 & $=(\$ H \$ 1) /((A 9) \wedge \$ H \$ 2)$ & $=C 9+\$ H \$ 8$ & $=C 9-\$ H \$ 8$ & & HYBRID & $=100^{*}\left(\operatorname{SUM}\left(\left((\mathrm{B} 2: \mathrm{B} 11-\mathrm{C} 2: \mathrm{C} 11)^{\wedge} 2\right) /(\mathrm{B} 2: \mathrm{B} 11)\right)\right) /(\operatorname{COUNT}(\mathrm{B} 2: \mathrm{B} 11)-1)$ \\
\hline 4 & 6.28562285138119 & $=(\$ H \$ 1) /((A 10) \wedge \$ H 2)$ & $=\mathrm{C} 10+\$ \mathrm{H} \$ 8$ & $=\mathrm{C} 10-\$ \mathrm{H} \$ 8$ & & NSD & $=100^{*}\left(\operatorname{SQRT}(\mathrm{SUM})\left(((\mathrm{B} 2: \mathrm{B} 11-\mathrm{C} 2: \mathrm{C} 11) /(\mathrm{B} 2: \mathrm{B} 11))^{\wedge} 2\right) /(\operatorname{COUNT}(\mathrm{B} 2: \mathrm{B} 11)-1)\right)$ \\
\hline 5.333 & 4.71451179552312 & $=(\$ H \$ 1) /\left((A 11)^{\wedge} \$ H \$ 2\right)$ & $=\mathrm{C} 11+\$ \mathrm{H} \$ 8$ & $=\mathrm{C} 11-\$ H \$ 8$ & & RMSE & $=\operatorname{SQRT}\left(\mathrm{SUM}\left((\mathrm{B} 2: \mathrm{B} 11-\mathrm{C} 2: \mathrm{C} 11)^{\wedge} 2\right) /(\operatorname{COUNT}(\mathrm{B} 2: \mathrm{B} 11)-1)\right)$ \\
\hline
\end{tabular}

Table 9: Non-linear regression solver based on Kimijima equation

\begin{tabular}{|c|c|c|c|c|c|c|c|}
\hline A & B & C & D & $\mathrm{E}$ & $\mathrm{F}$ & G & H \\
\hline d & i & KIMIJIMA EQUATION & Upper Cl & Lower Cl & & a & 4.83512327922962 \\
\hline 0.0333 & 755.029771937681 & $=(\$ H \$ 1) /(((A 2) \wedge \$+\$ 10)+\$ H \$ 2)$ & $=\mathrm{C} 2+\$ \mathrm{H} \$ 8$ & $=C 2-\$ H \$ \$ 8$ & & b & 0.00898367934147887 \\
\hline 0.0833 & 301.830629117945 & $=(\$ H \$ 1) /\left(\left((A 3)^{\wedge} \$ H \$ 10\right)+\$ H \$ 2\right)$ & $=\mathrm{C} 3+\$ \mathrm{H} \$ 8$ & $=C 3-\$ H \$ 8$ & & Mean of $i$ & =AVERAGE(B2:B11) \\
\hline 0.1667 & 150.824783476453 & $=(\$ H \$ 1) /\left(\left((A 4)^{\wedge} \$ H \$ 10\right)+\$ H \$ 2\right)$ & $=C 4+\$ H \$ 8$ & $=C 4-\$ H \$ 8$ & & df & $=\operatorname{COUNT}(\mathrm{B} 2: \mathrm{B} 11)-\operatorname{COUNT}(\mathrm{H} 1: \mathrm{H} 2)$ \\
\hline 0.25 & 100.569965622099 & $=(\$ H \$ 1) /\left(\left((A 5)^{\wedge} \$ H \$ 10\right)+\$ H \$ 2\right)$ & $=\mathrm{C} 5+\$ \mathrm{H} \$ 8$ & $=C 5-\$ H \$ 8$ & & SSE & $=$ SQRT(SUM((B2:B11-C2:C11)^2)/H4) \\
\hline 0.5 & 50.2849828110496 & $=(\$ H 1) /(((A 6) \wedge \$ H 10)+\$ H \$ 2)$ & $=C 6+\$ H \$ 8$ & $=C 6-\$ H \$ 8$ & & $R^{2}$ & $=1-\operatorname{SUM}\left((\mathrm{B} 2: \mathrm{B} 11-\mathrm{C} 2: \mathrm{C} 11)^{\wedge} 2\right) / \operatorname{SUM}\left((\mathrm{B} 2: B 11-\mathrm{H} 3)^{\wedge} 2\right)$ \\
\hline 1 & 25.1424914055248 & $=(\$ H \$ 1) /\left(\left((A 7)^{\wedge} \$ H \$ 10\right)+\$ H \$ 2\right)$ & $=\mathrm{C} 7+\$ \mathrm{H} \$ 8$ & $=\mathrm{C} 7-\$ \mathrm{H} \$ \$$ & & Critical t & $=\operatorname{TINV}(0.05, \mathrm{H} 4)$ \\
\hline 2 & 12.5712457027624 & $=(\$ H \$ 1) /(((A 8) \wedge \$ H 10)+\$ H \$ 2)$ & $=C 8+\$ H \$ 8$ & $=C 8-\$ H \$ 8$ & & $\mathrm{Cl}$ & $=\left(H 7^{*} H 5\right)$ \\
\hline 3 & 8.38083046850826 & $=(\$ H \$ 1) /(((A 9) \wedge \$ H 10)+\$ H \$ 2)$ & $=C 9+\$ H \$ 8$ & $=$ C9-\$H\$8 & & HYBRID & $\left.=100^{*}(\mathrm{SUM})\left(\left((\mathrm{B} 2: \mathrm{B} 11-\mathrm{C} 2: \mathrm{C} 11)^{\wedge} 2\right) /(\mathrm{B} 2: \mathrm{B} 11)\right)\right) /(\operatorname{COUNT}(\mathrm{B} 2: \mathrm{B} 11)-1)$ \\
\hline 4 & 6.28562285138119 & $=(\$ H \$ 1) /(((A 10) \wedge \$ H 10)+\$ H \$ 2)$ & $=\mathrm{C} 10+\$ \mathrm{H} \$ 8$ & $=\mathrm{C} 10-\$ \mathrm{H} \$ 8$ & & e & 0.00983506846736382 \\
\hline 5.333 & 4.71451179552312 & $=(\$ H \$ 1) /\left(\left((A 11)^{\wedge} \$ H \$ 10\right)+\$ H \$ 2\right)$ & $=C 11+\$ H \$ 8$ & $=C 11-\$ H \$ 8$ & & RMSE & $=$ SQRT(SUM((B2:B11-C2:C11)^2)/(COUNT(B2:B11)-1)) \\
\hline
\end{tabular}

Table 10: Non-linear regression solver based on Sherman equation 


\begin{tabular}{|c|c|c|c|c|c|c|c|}
\hline A & B & $\mathrm{C}$ & D & E & $\mathrm{F}$ & G & H \\
\hline d & i & SHERMAN EQUATION & Upper Cl & Lower Cl & & a & 1 \\
\hline 0.0333 & 755.029771937681 & $=(\$ H \$ 1) /((A 2+\$ H \$ 2) \wedge H \$ 10)$ & $=\mathrm{C} 2+\$ \mathrm{H} \$ \mathrm{~B}$ & $=C 2-\$ H \$ B$ & & b & 1 \\
\hline 0.0833 & 301.830629117945 & $=(\$ H \$ 1) /((A 3+\$ H \$ 2) \wedge H \$ 10)$ & $=C 3+\$+\$ S B$ & $=C 3-\$ 1 \$ S$ & & Mean of $\mathrm{i}$ & =AVERAGE(B2:B11) \\
\hline 0.1667 & 150.824783476453 & $=(\$ H \$ 1) /((A 4+\$ H \$ 2) \wedge H \$ 10)$ & $=C 4+\$ H \$ B$ & $=C 4-\$ H \$ B$ & & df & $=\operatorname{COUNT}(\mathrm{B} 2: \mathrm{B} 11)-\operatorname{COUNT}(\mathrm{H1}: \mathrm{H2})$ \\
\hline 0.25 & 100.569965622099 & $=(\$ H \$ 1) /((A 5+\$ H \$ 2) \wedge$ N\$ 10$)$ & $=\mathrm{C} 5+\$ \mathrm{H} \$ \mathrm{~B}$ & $=C 5-\$ H \$ B$ & & SSE & $=S Q R T\left(S U M\left((B 2: B 11-C 2: C 11)^{\wedge} 2\right) / H 4\right)$ \\
\hline 0.5 & 50.2849828110496 & $=(\$ H \$ 1) /((A 6+\$ H \$ 2) \wedge \$ \$ 10)$ & $=\mathrm{C} 6+\$ \mathrm{H} \$ \mathrm{8}$ & $=C 6-\$ H \$ 8$ & & $R^{2}$ & $=1-\mathrm{SUM}\left((\mathrm{B2}: \mathrm{B} 11-\mathrm{C} 2: \mathrm{C} 11)^{\wedge} 2\right) / \mathrm{SUM}\left((\mathrm{B} 2: B 11-\mathrm{H} 3)^{\wedge} 2\right)$ \\
\hline 1 & 25.1424914055248 & $=(\$ H \$ 1) /((A 7+\$ H \$ 2) \wedge H \$ 10)$ & $=\mathrm{C} 7+\$ \mathrm{H} \$ \mathrm{~B}$ & $=C 7-\$ H \$ 8$ & & Criticalt & $=\operatorname{TINV}(0.05, \mathrm{H} 4)$ \\
\hline 2 & 12.5712457027624 & $=(\$ H \$ 1) /((A 8+\$ H \$ 2) \wedge H \$ 10)$ & $=C 8+\$ H \$ 8$ & $=C 8-\$ H \$ 8$ & & $\mathrm{Cl}$ & $=\left(H 7^{*} H 5\right)$ \\
\hline 3 & 8.38083046850826 & $=(\$ H \$ 1) /((A 9+\$ H \$ 2) \wedge H \$ 10)$ & $=C 9+\$ H \$ 8$ & $=C 9-\$ H \$ 8$ & & HYBRID & $=100^{*}\left(\operatorname{SUM}\left(\left((B 2: B 11-C 2: C 11)^{\wedge} 2\right) /(B 2: B 11)\right)\right) /(\operatorname{COUNT}(B 2: B 11)-1)$ \\
\hline 4 & 6.28562285138119 & $=(\$ H \$ 1) /((A 10+\$ H \$ 2) \wedge H \$ 10)$ & $=\mathrm{C} 10+\$ \mathrm{H} \$ 8$ & $=\mathrm{C} 10-\$ \mathrm{H} \$ 8$ & & e & 1 \\
\hline 5.333 & 4.71451179552312 & $=(\$ H \$ 1) /((A 11+\$ H \$ 2) \wedge H \$ 10)$ & $=C 11+\$ \$ \$ 8$ & $=\mathrm{C} 11-\$ \mathrm{H} \$ 8$ & & RMSE & $=$ SQRT(SUM $\left.\left((B 2: B 11-C 2: C 11)^{\wedge} 2\right) /(\operatorname{COUNT}(B 2: B 11)-1)\right)$ \\
\hline
\end{tabular}

The non-linear regression solver was applied to estimate the parameters of the four empirical IDF equations that were used to represent intensity-duration relationships. The value of the constants corresponding to a minimum root mean square error (RMSE) between the computed rainfall intensity and the corresponding return period were selected as the exact value. Results of the estimated parameters are presented in Tables 11, 12, 13, 14, 15, 16, 17 and 18 representing Lokoja and Ilorin respectively.

Table 11: Estimated parameters of Talbot equation (Ilorin)

\begin{tabular}{|c|c|c|c|c|}
\hline $\begin{array}{l}\text { Return Periods } \\
\mathrm{T} \text { (Years) }\end{array}$ & $a$ & $\mathrm{~b}$ & $\begin{array}{l}\text { Minimized RMSE } \\
\left(\mathrm{OF}^{\mathrm{a}}\right)\end{array}$ & $\begin{array}{l}\text { Sum of Minimized Error } \\
\text { (SME) }\end{array}$ \\
\hline 2 & 25.181 & 0.00809 & $3.87622 \mathrm{E}-09$ & \multirow{6}{*}{$1.32170 \mathrm{E}-07$} \\
\hline 5 & 32.2234 & 0.00715 & $8.38749 \mathrm{E}-09$ & \\
\hline 10 & 36.8632 & 0.003398 & $2.55666 \mathrm{E}-08$ & \\
\hline 25 & 42.7578 & 0.00383 & $8.78360 \mathrm{E}-08$ & \\
\hline 50 & 47.5155 & 0.004765 & $6.00059 \mathrm{E}-09$ & \\
\hline 100 & 51.6486 & 0.002266 & $5.03231 \mathrm{E}-10$ & \\
\hline
\end{tabular}

Table 12: Estimated parameters of Bernard equation (Ilorin)

\begin{tabular}{|c|c|c|c|c|}
\hline $\begin{array}{l}\text { Return Periods } \\
\mathrm{T} \text { (Years) }\end{array}$ & $\mathrm{a}$ & $\mathrm{e}$ & $\begin{array}{l}\text { Minimized RMSE } \\
\left(\mathrm{OF}^{\mathrm{a}}\right)\end{array}$ & $\begin{array}{l}\text { Sum of Minimized Error } \\
\text { (SME) }\end{array}$ \\
\hline 2 & 4.73964 & 0.003176 & $1.21257 \mathrm{E}-07$ & \multirow{6}{*}{ 4.90162E-07 } \\
\hline 5 & 6.95028 & 0.05267 & $1.09076 \mathrm{E}-08$ & \\
\hline 10 & 6.92913 & 0.00183 & $4.17068 \mathrm{E}-08$ & \\
\hline 25 & 8.01204 & $1.465 \mathrm{E}-05$ & $1.94787 \mathrm{E}-07$ & \\
\hline 50 & 8.84136 & 0.000713 & $1.06478 \mathrm{E}-07$ & \\
\hline 100 & 10.18342 & 0.032529 & $1.50257 \mathrm{E}-08$ & \\
\hline
\end{tabular}

Table 13: Estimated parameters of Kimijima equation (Ilorin)

\begin{tabular}{|c|c|c|c|c|c|}
\hline $\begin{array}{l}\text { Return Periods } \\
\mathrm{T} \text { (Years) }\end{array}$ & $\mathrm{a}$ & $\mathrm{b}$ & $\mathrm{e}$ & $\begin{array}{l}\text { Minimized } \\
\text { RMSE } \\
\left(\mathrm{OF}^{\mathrm{a}}\right)\end{array}$ & $\begin{array}{l}\text { Sum of Minimized Error } \\
\text { (SME) }\end{array}$ \\
\hline 2 & 4.83512 & 0.008984 & 0.009835 & $4.12099 \mathrm{E}-08$ & \multirow{6}{*}{$1.08253646 \mathrm{E}-06$} \\
\hline 5 & 6.21772 & 0.013150 & 0.010228 & $9.57006 \mathrm{E}-09$ & \\
\hline 10 & 6.98678 & 0.00775 & 0.00219 & $2.21864 \mathrm{E}-07$ & \\
\hline 25 & 8.03047 & 0.000672 & 0.000987 & $1.87725 \mathrm{E}-08$ & \\
\hline 50 & 8.83466 & 0.000427 & $5.039 \mathrm{E}-06$ & $2.2828 \mathrm{E}-07$ & \\
\hline 100 & 10.28963 & 0.03746 & 0.017377 & $5.6284 \mathrm{E}-07$ & \\
\hline
\end{tabular}

Table 14: Estimated parameters of Sherman equation (Ilorin)

\begin{tabular}{|c|c|c|c|c|c|}
\hline $\begin{array}{l}\text { Return Periods } \\
\mathrm{T} \text { (Years) }\end{array}$ & $\mathrm{a}$ & $\mathrm{b}$ & $\mathrm{e}$ & $\begin{array}{l}\text { Minimized RMSE } \\
\left(\mathrm{OF}^{\mathrm{a}}\right)\end{array}$ & $\begin{array}{l}\text { Sum of Minimized } \\
\text { Error (SME) }\end{array}$ \\
\hline 2 & 4.7171 & 0.91445 & 0.000295 & $2.50979 \mathrm{E}-08$ & \multirow{6}{*}{$1.3948737 \mathrm{E}-06$} \\
\hline 5 & 6.43554 & 0.91334 & 0.03515 & $9.9194 \mathrm{E}-09$ & \\
\hline 10 & 7.23422 & 0.91419 & 0.025194 & $2.11926 \mathrm{E}-07$ & \\
\hline 25 & 8.01756 & 0.91445 & 0.0003895 & $8.85173 \mathrm{E}-08$ & \\
\hline 50 & 8.83718 & 0.914448 & 0.0003931 & $1.06131 \mathrm{E}-06$ & \\
\hline 100 & 10.48347 & 0.913803 & 0.045572 & $1.10314 \mathrm{E}-09$ & \\
\hline
\end{tabular}


Table 15: Estimated parameters of Talbot equation (Lokoja)

\begin{tabular}{|l|l|l|l|l|}
\hline $\begin{array}{l}\text { Return Periods } \\
\text { T (Years) }\end{array}$ & $\mathrm{a}$ & $\mathrm{b}$ & $\begin{array}{l}\text { Minimized RMSE } \\
\left(\mathrm{OF}^{\mathrm{a}}\right)\end{array}$ & $\begin{array}{l}\text { Sum of Minimized Error } \\
(\mathrm{SME})\end{array}$ \\
\hline 2 & 25.2447 & 0.010117 & $1.17022 \mathrm{E}-08$ & \multirow{3}{*}{$8.953636 \mathrm{E}-08$} \\
\hline 5 & 31.9614 & 0.01453 & $3.55266 \mathrm{E}-09$ & \\
\cline { 1 - 4 } & 36.30716 & 0.001613 & $3.51542 \mathrm{E}-08$ & \\
\cline { 1 - 4 } 50 & 41.88335 & $9.49 \mathrm{E}-05$ & $1.22965 \mathrm{E}-08$ & \\
\hline 100 & 46.40096 & $4.33 \mathrm{E}-05$ & $1.42290 \mathrm{E}-08$ & \\
\hline
\end{tabular}

Table 16: Estimated parameters of Bernard equation (Lokoja)

\begin{tabular}{|l|l|l|l|l|}
\hline $\begin{array}{l}\text { Return Periods } \\
\mathrm{T}(\text { Years })\end{array}$ & $\mathrm{a}$ & $\mathrm{e}$ & $\begin{array}{l}\text { Minimized RMSE } \\
\left(\mathrm{OF}^{\mathrm{a}}\right)\end{array}$ & $\begin{array}{l}\text { Sum of Minimized Error } \\
(\mathrm{SME})\end{array}$ \\
\hline 2 & 4.74632 & 0.00273 & $3.23766 \mathrm{E}-08$ & \multirow{2}{*}{$2.795093 \mathrm{E}-06$} \\
\hline 5 & 6.511715 & 0.051198 & $4.91264 \mathrm{E}-07$ & \\
\hline 10 & 6.81525 & 0.000815 & $2.36245 \mathrm{E}-09$ & \\
\cline { 1 - 4 } 25 & 7.89531 & 0.0031732 & $8.79262 \mathrm{E}-08$ & \\
\cline { 1 - 4 } 100 & 8.644688 & 0.000975 & $1.92134 \mathrm{E}-08$ & \\
\hline
\end{tabular}

Table 17: Estimated parameters of Kimijima equation (Lokoja)

\begin{tabular}{|l|l|l|l|l|l|}
\hline $\begin{array}{l}\text { Return Periods } \\
\text { T (Years) }\end{array}$ & $\mathrm{a}$ & $\mathrm{b}$ & $\mathrm{e}$ & $\begin{array}{l}\text { Minimized RMSE } \\
\left(\mathrm{OF}^{\mathrm{a}}\right)\end{array}$ & $\begin{array}{l}\text { Sum of Minimized Error } \\
(\mathrm{SME})\end{array}$ \\
\hline 2 & 4.83554 & 0.009896 & 0.008047 & $6.14915 \mathrm{E}-09$ & \\
\cline { 1 - 5 } 2 & 6.07776 & 0.00465 & 0.007258 & $3.73719 \mathrm{E}-07$ & \\
\hline 10 & 6.97995 & 0.009022 & 0.0098017 & $4,70746 \mathrm{E}-07$ & \\
\hline 25 & 7.94581 & 0.011668 & $5.2597 \mathrm{E}-05$ & $6.85129 \mathrm{E}-07$ & \\
\hline 50 & 8.63231 & $6.236 \mathrm{E}-05$ & $8.2038 \mathrm{E}-05$ & $2.36767 \mathrm{E}-07$ & \\
\hline 100 & 9.79126 & 0.021345 & 0.011866 & $9.28113 \mathrm{E}-07$ & \\
\hline
\end{tabular}

Table 18: Estimated parameters of Sherman equation (Lokoja)

\begin{tabular}{|c|c|c|c|c|c|}
\hline $\begin{array}{l}\text { Return Periods } \\
\mathrm{T} \text { (Years) }\end{array}$ & $\mathrm{a}$ & $\mathrm{b}$ & $\mathrm{E}$ & $\begin{array}{l}\text { Minimized } \\
\text { RMSE } \\
\left(\mathrm{OF}^{\mathrm{a}}\right)\end{array}$ & $\begin{array}{l}\text { Sum of Minimized } \\
\text { Error (SME) }\end{array}$ \\
\hline 2 & 4.72472 & 0.91445 & $1.27276 \mathrm{E}-06$ & 6.74459E-08 & \multirow{6}{*}{$1.3681693 \mathrm{E}-06$} \\
\hline 5 & 6.42970 & 0.913507 & 0.039861 & $1.60538 \mathrm{E}-08$ & \\
\hline 10 & 6.80779 & 0.91445 & 0.0001464 & $5.74636 \mathrm{E}-08$ & \\
\hline 25 & 7.85796 & 0.914449 & 0.0003113 & $1.04931 \mathrm{E}-06$ & \\
\hline 50 & 8.63145 & 0.91445 & $5.45999 \mathrm{E}-05$ & $1.72538 \mathrm{E}-07$ & \\
\hline 100 & 10.03473 & 0.91332 & 0.035553 & $5.35803 \mathrm{E}-09$ & \\
\hline
\end{tabular}

The regional IDF formula parameters were mostly generated for ungauged areas to estimate rainfall intensity for various return period and rainfall duration. Using 40 years annual maximum daily rainfall data (1974 - 2013), for two locations within the lower Niger River Basin (Ilorin and Lokoja), the parameters of Talbot, Bernard, Kimijima and Sherman equations were estimated based on the minimum root mean square error (RMSE) using the non-linear regression solver. To select the model that best fit each location, the sum of minimized error was employed and the model with the least sum of minimized error was selected as the best fit model. Based on the results of Tables 11, 12, 13 and 14, it was observed that Talbot equation had the least sum of minimized error of $1.32170 \mathrm{E}-07$ and was declared the best fit model for Ilorin. Using the Talbot model, the following equations were generated for Ilorin in order to estimate the rainfall intensity.

For 2 years return period;

For 5 years return period;

For 10 years return period;

For 25 years return period;

$$
\begin{aligned}
& i=\frac{25.181}{d+0.00809} \\
& i=\frac{32.2234}{d+0.00715} \\
& i=\frac{36.8632}{d+0.003398} \\
& i=\frac{42.7578}{d+0.00382}
\end{aligned}
$$


For 50 years return period;

For 100 years return period;

$$
i=\frac{47.5155}{d+0.004765}
$$

$$
i=\frac{51.6486}{d+0.002266}
$$

More also, based on the results of Tables 15, 16, 17 and 18, it was observed that Talbot equation had the least sum of minimized error of 8.953636E-08 and was again declared the best fit model for Lokoja. Using the Talbot model, the following equations were generated for Lokoja in order to estimate the rainfall intensity.

For 2 years return period;

$$
\begin{aligned}
& i=\frac{25.2447}{d+0.010117} \\
& i=\frac{31.9614}{d+0.01453} \\
& i=\frac{36.30716}{d+0.001613}
\end{aligned}
$$

For 5 years return period;

For 10 years return period;

For 25 years return period;

$$
i=\frac{41.88335}{d+9.4876 E-05}
$$

For 50 years return period;

$$
\begin{aligned}
& i=\frac{46.40096}{d+4.3340 E-05} \\
& i=\frac{50.45183}{d+3.3096 E-05}
\end{aligned}
$$

Where; $d$ is duration (hrs). Based on the Talbot equation, the predicted rainfall intensity for Ilorin and Lokoja were computed and presented in Tables 19 and 20 respectively; while the IDF curves are presented in Figures 7 and 8 respectively.

Table 19: Predicted rainfall intensities for Ilorin using Talbot equation

\begin{tabular}{|l|l|l|l|l|l|l|}
\hline $\begin{array}{l}\text { Duration } \\
(\mathrm{hrs})\end{array}$ & $\begin{array}{l}\text { Intensity } \\
(\mathrm{mm} / \mathrm{hr}) \\
(\mathrm{T}=2 \mathrm{yrs})\end{array}$ & $\begin{array}{l}\text { Intensity } \\
(\mathrm{mm} / \mathrm{hr}) \\
(\mathrm{T}=5 \mathrm{yrs})\end{array}$ & $\begin{array}{l}\text { Intensity } \\
(\mathrm{mm} / \mathrm{hr}) \\
(\mathrm{T}=10 \mathrm{yrs})\end{array}$ & $\begin{array}{l}\text { Intensity } \\
(\mathrm{mm} / \mathrm{hr}) \\
(\mathrm{T}=25 \mathrm{yrs})\end{array}$ & $\begin{array}{l}\text { Intensity } \\
(\mathrm{mm} / \mathrm{hr}) \\
(\mathrm{T}=50 \mathrm{yrs})\end{array}$ & $\begin{array}{l}\text { Intensity } \\
(\mathrm{mm} / \mathrm{hr}) \\
(\mathrm{T}=100 \mathrm{yrs})\end{array}$ \\
\hline 0.0333 & 608.3836676 & 796.6229913 & 1107.006401 & 1151.880388 & 1248.272691 & 1452.190294 \\
\hline 0.0833 & 275.5334282 & 356.2564953 & 442.5388122 & 490.792011 & 539.5503321 & 603.6112475 \\
\hline 0.1667 & 144.0643057 & 185.35174 & 221.138371 & 250.7494722 & 277.1148631 & 305.6745144 \\
\hline 0.25 & 97.56674028 & 125.3097414 & 147.456198 & 168.4571744 & 186.5071733 & 204.7386489 \\
\hline 0.5 & 49.5601173 & 63.53820369 & 73.729798 & 84.86721448 & 94.1339039 & 102.8311691 \\
\hline 1 & 24.97892053 & 31.99463834 & 36.866598 & 42.59508677 & 47.29016238 & 51.53182888 \\
\hline 2 & 12.5397766 & 16.05430586 & 18.434998 & 21.33814414 & 23.7012817 & 25.79507418 \\
\hline 3 & 8.37109262 & 10.7155945 & 12.29113133 & 14.23447477 & 15.81338308 & 17.20320585 \\
\hline 4 & 6.282543556 & 8.041475862 & 9.219198 & 10.67925131 & 11.86474113 & 12.90483941 \\
\hline 5.333 & 4.714580732 & 6.03417507 & 6.915680018 & 8.011849753 & 8.901759444 & 9.680604491 \\
\hline
\end{tabular}

Table 20: Predicted rainfall intensities for Lokoja using Talbot equation

\begin{tabular}{|l|l|l|l|l|l|l|}
\hline $\begin{array}{l}\text { Duration } \\
(\mathrm{hrs})\end{array}$ & $\begin{array}{l}\text { Intensity } \\
(\mathrm{mm} / \mathrm{hr}) \\
(\mathrm{T}=2 \mathrm{yrs})\end{array}$ & $\begin{array}{l}\text { Intensity } \\
(\mathrm{mm} / \mathrm{hr}) \\
(\mathrm{T}=5 \mathrm{yrs})\end{array}$ & $\begin{array}{l}\text { Intensity } \\
(\mathrm{mm} / \mathrm{hr}) \\
(\mathrm{T}=10 \mathrm{yrs})\end{array}$ & $\begin{array}{l}\text { Intensity } \\
(\mathrm{mm} / \mathrm{hr}) \\
(\mathrm{T}=25 \mathrm{yrs})\end{array}$ & $\begin{array}{l}\text { Intensity } \\
(\mathrm{mm} / \mathrm{hr}) \\
(\mathrm{T}=50 \mathrm{yrs})\end{array}$ & $\begin{array}{l}\text { Intensity } \\
(\mathrm{mm} / \mathrm{hr}) \\
(\mathrm{T}=100 \mathrm{yrs})\end{array}$ \\
\hline 0.0333 & 581.4473593 & 668.2291449 & 1090.306718 & 1254.184923 & 1391.611038 & 1514.039696 \\
\hline 0.0833 & 270.2366807 & 326.7034652 & 435.8618771 & 502.2292976 & 556.7446661 & 605.4995124 \\
\hline 0.1667 & 142.7730365 & 176.3582188 & 217.8010131 & 251.1069345 & 278.2777411 & 302.6093154 \\
\hline 0.25 & 97.0513269 & 120.8233471 & 145.230253 & 167.4698445 & 185.5716693 & 201.7890298 \\
\hline 0.5 & 49.48805862 & 62.11766078 & 72.615933 & 83.75080812 & 92.79387663 & 100.8990873 \\
\hline 1 & 24.99185738 & 31.50365194 & 36.308773 & 41.87937665 & 46.39894907 & 50.45068679 \\
\hline 2 & 12.5588212 & 15.8654376 & 18.155193 & 20.94068162 & 23.19997726 & 25.22562919 \\
\hline 3 & 8.38661753 & 10.60244881 & 12.10399967 & 13.96067516 & 15.46676322 & 16.81714964 \\
\hline 4 & 6.295252732 & 7.961430105 & 9.078403 & 10.47058915 & 11.60011431 & 12.61288605 \\
\hline 5.333 & 4.724714057 & 5.976852865 & 6.809631001 & 7.85347926 & 8.700653087 & 9.460269198 \\
\hline
\end{tabular}




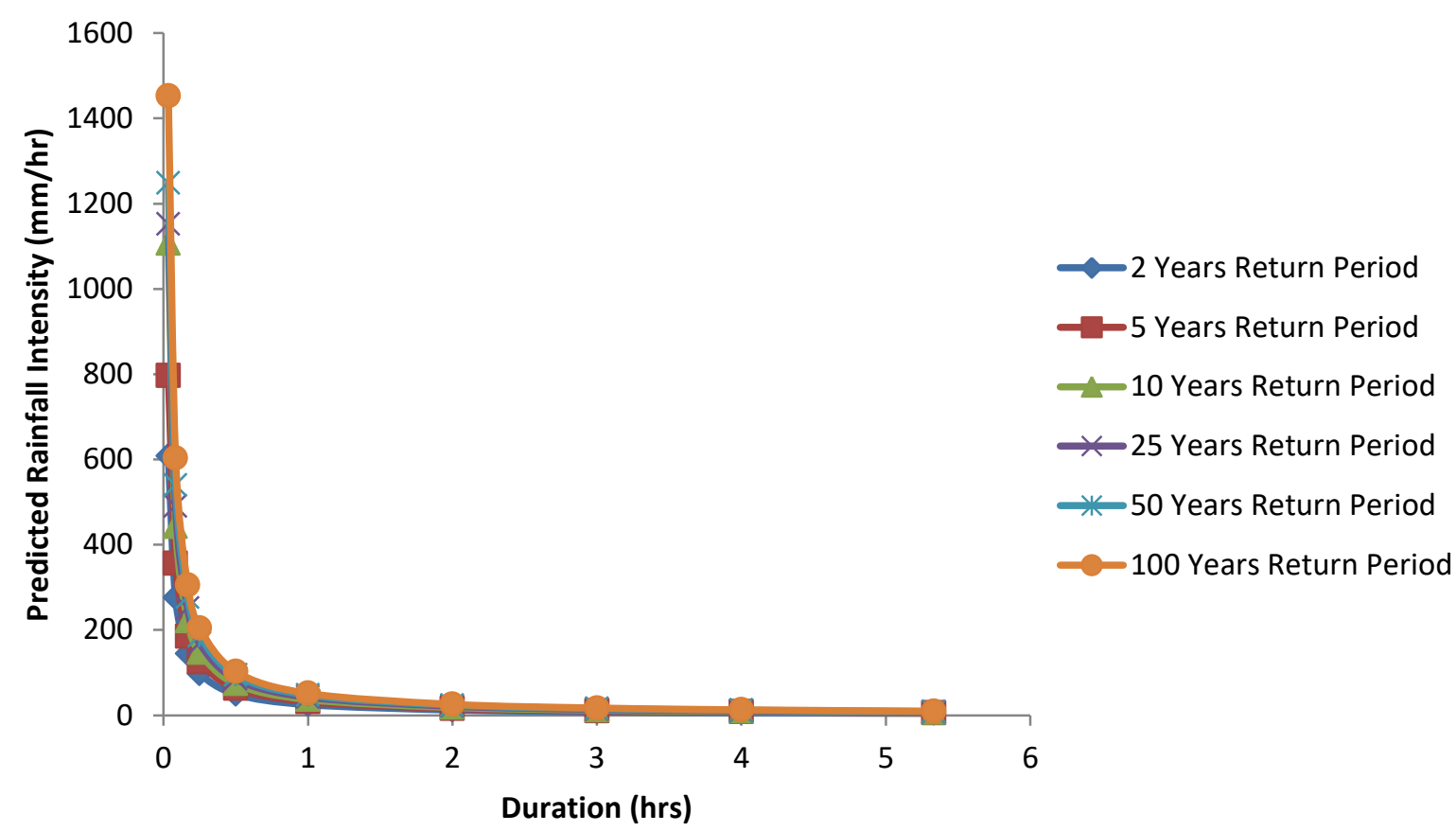

Figure 7: IDF curves for Ilorin using Talbot equation

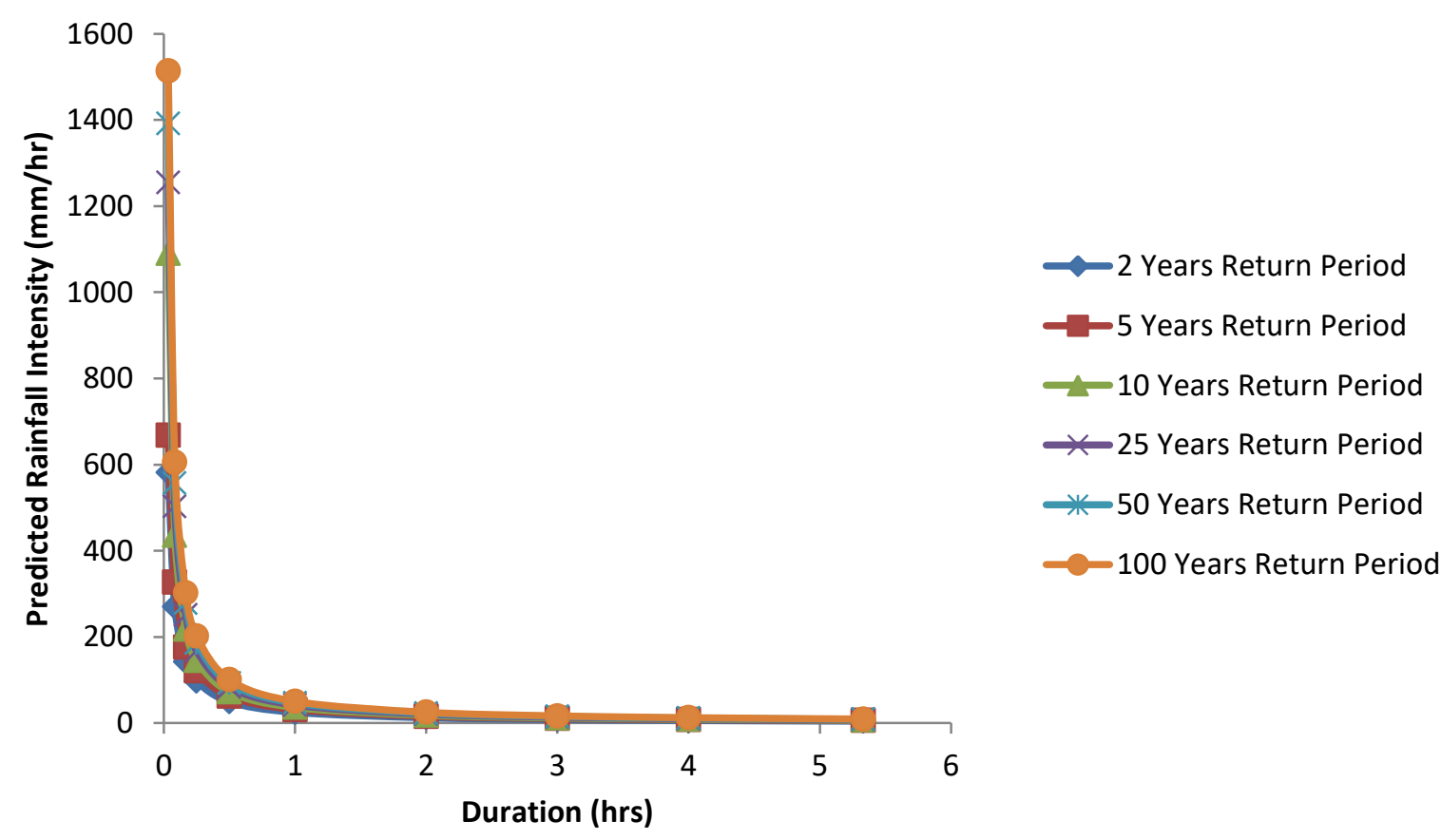

Figure 8: IDF curves for Lokoja using Talbot equation

\subsection{Conclusions}

The data used in this study represent annual daily rainfall data obtained from Nigerian Meteorological Agency, NIMET. In the study, descriptive statistics were used to describe the basic features of the data. Homogeneity test was carried out to establish the fact that the data used comes from the same population distribution. Outliers were identified and removed using the labeling rule before statistical analysis was carried out. Rainfall intensity duration curves were generated from the plot of rainfall intensities against duration for corresponding return periods.

Results of the homogeneity tests carried put on the annual maximum daily rainfall season for Lokoja and Ilorin revealed that the data used are statistically homogeneous. After removal of outliers, Gumbel probability distribution model was used in calculating the rainfall intensity for the two stations based on the selected Return Periods. From the computed rainfall intensity, IDF curves were generated. Intensity Duration Frequency data are needed by hydrologists and Engineers during the planning and design of water resource projects. The achievement of a set of empirical equations for 
rainfall intensity prediction is of importance in hydrology for various applications including Flood Frequency Analysis and Flood Prediction and Management.

\section{References}

Abam, T (2001); Regional hydrological research perspectives in the Niger Delta, Hydrological Science Journal, 461, pp. 13-25

Adeaga, O., Gil, M., Claudine, D., Elbaz-poulichet, F., Nathalie, R., Jean-luc, S. and Eric, S. (2012); Rainfall-Runoff Simulation in Part of Lower Niger Basin, Journal of Environmental Science and Engineering, 1, pp. 812-819.

Antigha, R. E. and Ogharekpe, N. M. (2013); Development of intensity duration frequency curve for Calabar Metropolis, south- south Nigeria. International Journal of Engineering and Science (IJES), 2, pp. $39-42$

Bell, F. C. (1969); generalized rainfall duration frequency relationships, Journal of Hydraulic Div., ASCE, 95(1), pp. 311-327.

Bernard, M. M. (1932); Formulas for rainfall intensities of long duration, Transactions, ASCE, 96 (Paper No.1801), pp. 592-624

Cardoso, C. O., Idelgardis, B., Olívio, J. S. and Carlos Augusto de Paiva, S. (2013); Generation of Intensity Duration Frequency Curves and Intensity Temporal Variability Pattern of Intense Rainfall for Lages/SC. Brazilian Archives of Biology and Technology, pp. 1-10.

Chen, C. L. (1983); Rainfall intensity-duration -frequency formulas, Journal of Hydraulic Engineering, ASCE, 109(12), pp. 1603-1621

Chow, V. T., Maidment, D. R. and Mays, L. W. (1988); Applied Hydrology. McGraw-Hill International Editions, New York, USA

Clanet, J. C. and Ogilvie, A. (2009); Basin Focal Project Niger, CPWF project report series, Challenge Program on Water and Food, Colombo, Sri Lanka.

Dupont, B. S., Allen, D. L. and Clark, K. D. (2000); Revision of the Rainfall-Intensity-Duration Curves for the Commonwealth of Kentucky. Kentucky Transportation Center, College of Engineering, University of Kentucky Lexington, Kentucky

El-Sayed, E. A. (2011); Generation of Rainfall Intensity Duration Frequency Curves for Ungauged Sites. Nile Basin Water Science \& Engineering Journal, 4(1), pp. 112-124.

Hershfield, D. M. (1961); Estimating the Probable Maximum precipitation, Journal of the Hydraulic Division, Proceeding of the ASCE, HY5, pp. 99 - 116.

Levi, D. B., Julie, E. K., Olsen, J. R., Pulwarty, R. S., Raff, D. A., et al. (2009). Climate Change and Water Resources Management: A Federal Perspective. Circular 1331, pp. 1 - 72.

McCuen, R. H. (2003); Modelling Hydrologic Change: Statistical Method. Lewis publishers, New York, 2nd edition, pp. 126-267

Prodanovic, P. and Simonovic, S. (2007); Development of Rainfall Intensity Duration Frequency Curve for the City of London under Changing Climate. Department of Civil and Environmental Engineering, London, Report (58)

Pruski, F. F., Silva, D. D., Teixeira, A. F., Silva, J. M. A., Cecílio, R. A. and Silva, D. F. (2002). Chuvas intensas para o Brasil. In: Congresso Brasileiro de Engenharia Agrícola, 31, 2002, Salvador. Anais. Salvador: Sociedade Brasileira de Engenharia Agrícola. 
Raes, D., Willens, P. and Gbaguidi (2006); Rainbow - A software package for analyzing data and testing the homogeneity of historical data sets, Vol. 1, pp. 1-15.

Ugbong, I. A. (2000); An Analysis of Runoff flow, Channel Characteristics, Flood and Erosion Menace in the Calabar Drainage Basin, M.Sc Thesis, Dept. of Geography and Regional Planning, University of Calabar

Cite this article as:

Ehiorobo J. O., Izinyon O. C. and Ilaboya R. O., 2020. Development of Intensity Duration Frequency (IDF) Curves for Rainfall Prediction within the Middle Niger River Basin, Nigeria. Nigerian Journal of Environmental Sciences and Technology, 4(2), pp. 382-397. https://doi.org/10.36263/nijest.2020.02.0231 